\title{
CEPHEIDS IN THE MAGELLANIC CLOUDS
}

\author{
JOHN A.R. CALDWELL, C. DAVID LANEY \\ South African Astronomical Observatory \\ PO Box 9, Observatory 7935, Cape \\ South Africa
}

\begin{abstract}
Cepheid variables provide a key to understanding the structure and distance of the Magellanic Clouds, as well as providing accurate reddenings there. Recent major observing programs have continually expanded the data base of photometry and velocities. It is important to try to reconcile the plethora of Cepheid data into a consonant picture of the three-dimensional spatial structure of the Clouds and to compare in a consistent manner the bulk Cloud distance moduli found by several photometric methods.
\end{abstract}

\section{Discovery, census and photometry}

Payne-Gaposchkin and Gaposchkin (1966) and Payne-Gaposchkin (1971) laid monumental foundations to the study of Cepheid variables in the Magellanic Clouds. They provided positions, periods, and photographic light curves for over 1100 in each Cloud. After a hiatus, new Cloud Cepheids are once again being discovered, including bright ones (e.g. Hodge \& Lee 1984, Kurochkin et al. 1989, van Genderen \& Hadiyanto Nitihardjo 1989, Mateo et al. 1990).

Madore (1985) has summarized the photometric (UBVRI, Iv, JHK) data base contributed by many workers up to 1985 . Caldwell and Laney (1990, hereafter CL) have collected and prepared a homogenized tabulation of the data cited by Madore and subsequent sources: Caldwell and Coulson (1984a, 1984b), Caldwell et al. (1986), Laney and Stobie (1986a, 1986b), Freedman et al. (1985), Mathewson et al. (1986, 1988), Mateo et al. (1990), Visvanathan (1985, 1989), Walker (1987b), Welch et al. (1987). Possibly less well known are the observations by Martin (1980, 1981) and Dean (1981), and the useful photoelectric light curve results in Martin et al. (1981). At present about 141 LMC and 186 SMC Cepheids have photometry published in the systems mentioned. These Cepheids cover the apparent bodies of both Clouds well (cf. LC), except for the LMC bar. Because of past observational selection, the fraction of Cloud Cepheids with some photometry is near unity for $\log P>1$, but decreases rapidly for shorter periods.

Besides the above-cited data, extensive photoelectric work also appeared in the Washington system (Harris 1981, 1983) and the Walraven system (van Genderen 1983a). Modern photographic photometry covering a much larger sample of Cepheids than is usually feasible in photoelectric work was provided by Martin et al. (1981) and Wayman et al. (1984) et op. cit.

\section{Interstellar reddening}

For periods longer than about 10 days, the best reddening determinations presently available for Cloud Cepheids come from BVI multicolour photometry. This is the case because the intrinsic line is narrow and well calibrated, the reddening line intersects the intrinsic line at a favourable angle, 
and relatively bright, uncrowded long-period Cloud Cepheids are rather amenable to obtaining accurate simultaneous B-V and V-I pairs. For periods shorter than about 10 days the BVI reddenings lose their salient advantages; the problem of individual reddenings for short-period Cloud Cepheids requires more investigation.

The available photometric material was reanalyzed for BVI reddening determinations in both Clouds, using principally Caldwell and Coulson (1985 a,b), Freedman et al. (1985), Caldwell et al. (1986), and Dean (1981). The LMC Cepheids yield 30 reddenings of probably good quality. They have a mean $\mathrm{E}(\mathrm{B}-\mathrm{V})=0.08$, median $=0.07$, and scatter of 0.04 . The SMC Cepheids yield 44 such reddenings, with mean and median $E(B-V)=0.06$, and scatter again of 0.04 . The scatters must encompass both the observational errors and the actual reddening scatter. BVI relative reddenings of Cloud Cepheids longer than 10 days are thus accurate to better than $0.04 \mathrm{mag}$, and we consider that 0.02 is a more likely figure for well-observed stars.

There are zones in each Cloud where one might well suspect higher reddenings to prevail. These are the regions in the central bars of each Cloud where Hodge $(1972,1974)$ has catalogued concentrations of dark nebulae. We have circumscribed these regions with elliptical fits (cf. LC). Then excluding "dark cloud zones," areal plots of the Cloud reddening determinations show that the values give a good representative sampling of positions across both Clouds. Also there is no angular segregation visible when the high and low halves of the reddening values are plotted separately.

Because of the small observed range of individual Cepheid reddenings, may one assume a statistical reddening value to apply to the rest of the Cepheids for purposes such as obtaining a PL distance to the Clouds? We can test this proposition by examining the period-colour relation in the Clouds. B-V is the best colour choice since it affords the most extensive sample of accurate phaseaveraged means. Let us also take the liberty henceforth of excluding periods outside the range 3$100 \mathrm{~d}$, obvious overtones, and a small number of Cepheids that appear abnormal (HV2301, HV2738 and HV 2749 in the LMC, and HV1369, HV1484, HV 1636 and HV1641 in the SMC). If we deredden all Cepheids of unknown reddening (dark cloud zones excluded) by the Cloud median reddening value, and define $\mathrm{DBV}$ as the magnitude average of $(\mathrm{B}-\mathrm{V}) \mathrm{O}-0.7$, and dlogP as $\log \mathrm{P}-1.0$, then the $\mathrm{B}-\mathrm{V}$ period colour relations in the Clouds are (cf. LC):

$$
\begin{aligned}
\mathrm{dBVo}= & 0.37 \mathrm{dlog} \mathrm{P}+0.02, \mathrm{~N}=89, \sigma=0.09(\mathrm{LMC}) \\
& \pm .03 \quad \pm .01
\end{aligned}
$$

and

$$
\begin{aligned}
& \mathrm{dBVo}=0.45 \text { dlogP }-0.12, \mathrm{~N}=73, \sigma=0.09(\mathrm{SMC}) \\
& \pm .03 \quad \pm .01
\end{aligned}
$$

By introducing as an additional free parameter an arbitrary colour offset between the knownreddening and statistical-reddening colours, one finds that in both Clouds the statistically dereddened colours appear consistent with or even slightly too blue compared with the individually dereddened colours. Hence the median reddening applied to the bulk of the Cepheids is surely not an underestimate of the actual reddenings.

The mean reddening values of 0.08 (LMC) and 0.06 (SMC) are in good agreement with the weighted average of the VBLUW reddenings of van Genderen (1983b), with the infrared colours of Cloud Cepheids as compared with solar neighbourhood giants and dwarfs (Laney \& Stobie 1986b), and with a thorough study of the BVRI colours of Cloud supergiants by Grieve and Madore (1986). The latter obtain mean reddenings of 0.10 (LMC) and 0.09 (SMC), but with apparently large observational (not necessarily intrinsic) spread. They too report no discernible areal trends. 


\section{Visual luminosity laws and cloud geometry}

CL plot up-to-date versions of the visual PL relations in the Clouds:

$$
\begin{gathered}
<\mathrm{Vo}>=-2.81 \mathrm{~d} \log \mathrm{P}+ \\
\pm .06 \quad 14.42, \mathrm{~N}=88, \sigma=0.21(\mathrm{LMC}) \\
\pm .02
\end{gathered}
$$

and

$$
\begin{gathered}
<\mathrm{Vo}>=-2.63 \mathrm{~d} \log \mathrm{P}+ \\
\pm .08 \quad 14.70, \mathrm{~N}=73, \sigma=0.23(\mathrm{SMC}) \\
\pm .03
\end{gathered}
$$

In these regressions a geometrical tilt of each Cloud out of the plane of the sky has been permitted so as to optimize the residuals; it is consistent with that found (with higher accuracy) from the PLC solution below. The statistically dereddened magnitudes are consistent in zero point with the individually dereddened ones, or possibly marginally too bright in the case of the LMC. Little can be inferred about the dark nebula zone in the LMC (i.e. the bar) given the lack of Cepheids in its interior that have measured V magnitudes. For the SMC dark nebula zones, including Cepheids within those boundaries in a PL solution implies a V zero point fainter by $0.32 \pm 0.07 \mathrm{mag}$ than determined from the remaining Cepheids. Clearly the statistical reddening assumption is not justified here, and these Cepheids (lacking individual reddenings) should be excluded.

It has occasionally been remarked that the instability strip in both Clouds narrows shortward of about $10 \mathrm{~d}$. CL find in this connection:

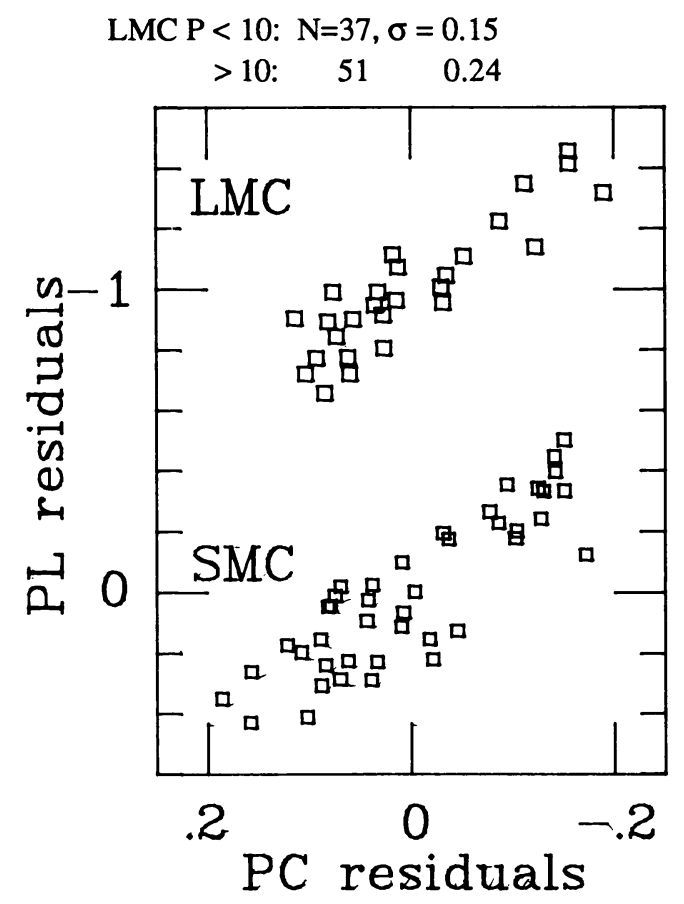

Figure 1. Correlation of $\langle\mathrm{Vo}\rangle$ residuals versus (B-V)o residuals at fixed period for Cloud Cepheids with known reddenings. 
If one plots (Fig. 1) the PL-residuals versus the PC-residuals for Cepheids with individually determined reddening, one obtains the well-known "colour term" correlation in both Clouds, which results from the temperature width of the instability strip at fixed period. Much discussion has taken place in the literature about the value of this slope, the worries arising because there are observational, and indeed correlated, uncertainties attached to both variables. An extreme view has been that observational errors on the relative individual reddenings are several times larger than believed by us to be the case, and that therefore the apparent relation is predominantly an artifact of observational scatter in the relative reddenings. Laney and Stobie (1986b) effectively ruled out this possibility by demonstrating in the infrared that the colour term is a physical property caused by the finite width of the strip; the infrared is useful in this regard because there the colour term and the reddening slope are entirely different.

A PLC relation which is to predict Cepheid magnitudes from their periods and colours must solve for the physically correct colour term BETA. Restricted to the Cloud Cepheids with individual reddenings, CL obtain:

LMC $(\mathrm{N}=27)$ BETA $>=2.19 \pm 0.23$ (least sqrs. with all error in mag.)

$=2.45 \pm 0.26$ (ridge line solution)

$<=2.75 \pm 0.29$ (least sqrs. with all error in colour).

SMC $(\mathrm{N}=42)$ BETA $>=2.34 \pm 0.19$ (least sqrs. with all error in mag.)

$=2.63 \pm 0.25$ (ridge line solution)

$<=3.35 \pm 0.43$ (least sqrs. with all error in colour).

While one may wish to dispute how to apportion the errors between the axes and account for subtle biases to the slope, we consider it not unreasonable just to adopt a ridge line type solution in each case, namely BETA $=2.5$ in the LMC and BETA $=2.6$ in the SMC, both with about \pm 0.3 . Applying the PLC colour term results in a greatly improved fit to the Cloud Cepheid magnitudes (cf. CL):

$$
\begin{aligned}
<\mathrm{Vo}>= & -3.72 \mathrm{~d} \log \mathrm{P}+2.5 \mathrm{dBV} 0+ \\
& \pm .04 \quad \pm .37, \mathrm{~N}=88, \sigma=0.13(\mathrm{LMC}) \\
& -0.033 \mathrm{dXI} / 10000-0.062 \mathrm{dETA} / 10000,
\end{aligned}
$$

and

$$
\begin{array}{cc}
<\mathrm{Vo}>= & -3.75 \mathrm{~d} \log \mathrm{P}+2.6 \mathrm{dBV0}+ \\
\pm .05 & 15.02, \mathrm{~N}=73, \sigma=0.14(\mathrm{SMC}) \\
\pm .02
\end{array}
$$

$-0.208 \mathrm{dXI} / 10000-0.032 \mathrm{dETA} / 10000$.

The angular coordinates correspond to the standard Wesselink (1959) ones as follows: (LMC) $\mathrm{dXI}=\mathrm{XI}+2260, \mathrm{dETA}=\mathrm{ETA}-941,(\mathrm{SMC}) \mathrm{dXI}=\mathrm{XI}-866, \mathrm{dETA}=\mathrm{ETA}+575$; these offsets normalize the angular coordinates to the centre of the Cepheid distributions. Thus we find a tilt of $34 \pm 8^{\circ}$ (or $0.026 \pm 0.007 \mathrm{mag} / \mathrm{deg}$ ) at position angle $208^{\circ}$ in the case of the LMC Cepheids and a tilt of $63 \pm 4^{\circ}$ (or $0.076 \pm .015 \mathrm{mag} / \mathrm{deg}$ ) at position angle $261^{\circ}$ in the case of the SMC. 
From the appearance of the Clouds on the sky it seems reasonable to model the LMC as a tilted disk, with $\mathrm{PA}=208^{\circ}$ and tilt $=34^{\circ}$, but the SMC does not have the symmetry of a simple disk tilted to PA $=261^{\circ}$. We examine the SMC structure implied by the PLC residuals by splitting the Cepheids into two subsets. Fig. 2. shows the "wing (plus N.E. bar) Cepheids." The dashed line is a wing axis defined by the Cepheids' loci and the direction to the LMC centre. Fig. 3 shows an alternative grouping, the "bar Cepheids." Here the dashed line is a bar axis defined by the Cepheids loci alone. By scaling both the angular and magnitude axes to distances in kpc, it is possible in Figs. 4 and 5 to represent what the Cepheids would imply about the depth structure of the SMC. We defer discussing this until after first examining the input from the infrared Cepheid data.

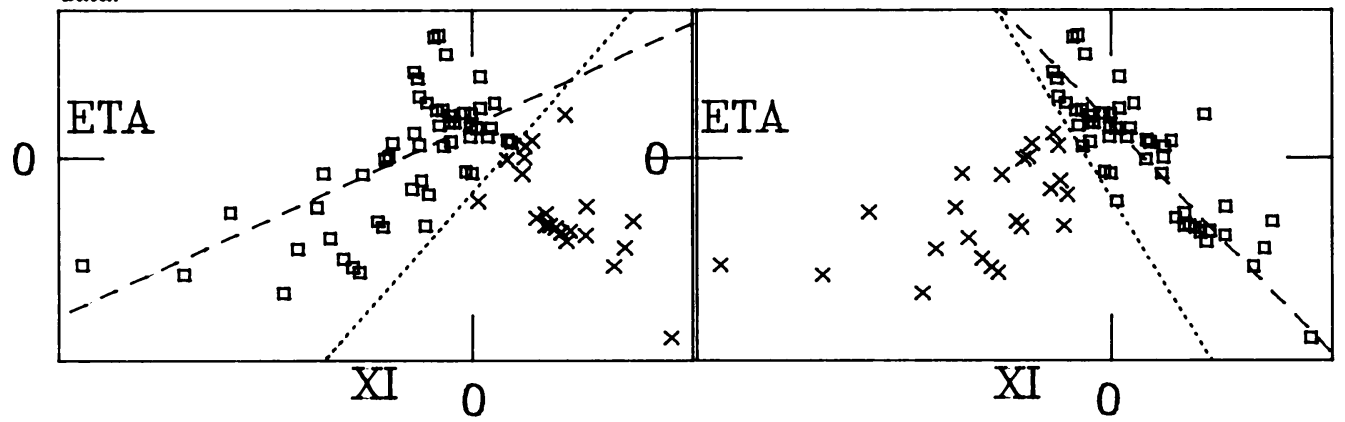

Figure 2. SMC wing (+N.E. bar) Cepheids defined by squares.

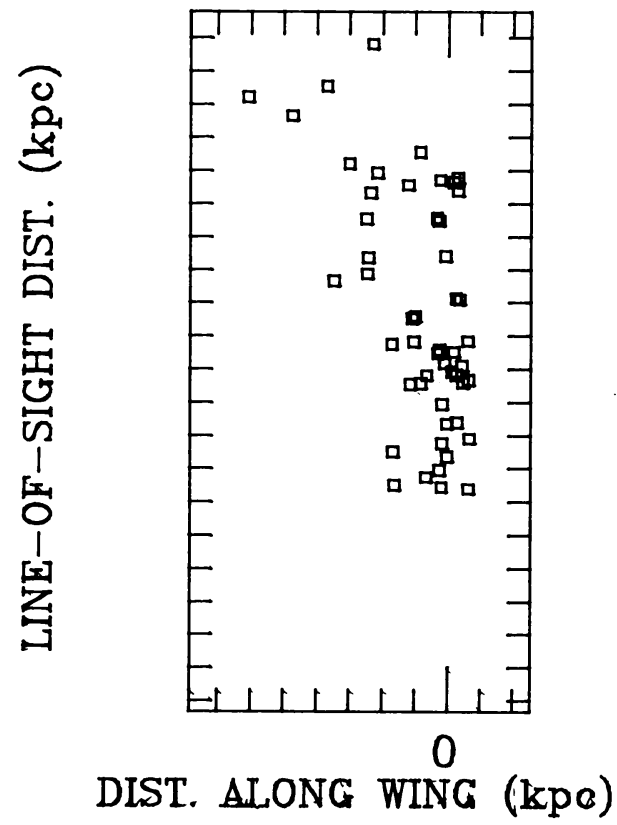

Figure 3. SMC bar Cepheids defined by squares.

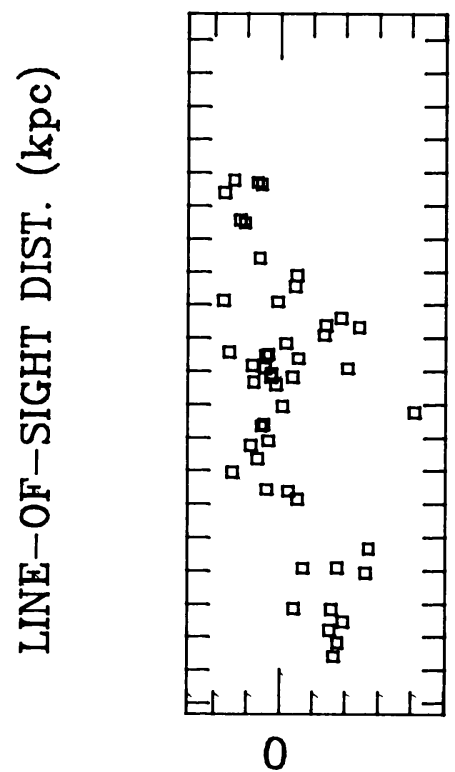

DIST, ALONG BAR (kpc)

Figures 4 (left) and 5 (right). SMC depth structure on wing and bar axes. 


\section{Infrared luminosity laws and cloud geometry}

Welch et al. (1987) have published few-phase JHK data on Clouds Cepheids and have included most (not all) of the full-phase JHK data from Laney and Stobie (1986a,b). We have retransformed and combined all this work onto the CTIO JHK system using the transformation equations of Glass (1985). Data with estimated errors above 0.1 because of corrections to mean light were culled. CL plot these versions of the infrared PL relations in the Clouds. They find that SMC Cepheids located in the dark cloud zones and lacking individual reddenings are too red in (J$\mathrm{K}) \mathrm{O}$ and too faint in Ho by $0.16 \pm 0.06 \mathrm{mag}$ if assigned the statistical median reddening. Thus excluding such Cepheids, CL obtain these PL-H relations in the Clouds:

and

$$
\begin{gathered}
<\mathrm{Ho}>= \\
\pm .07 \mathrm{~d} \quad \mathrm{~d} \log \mathrm{P}+12.83, \mathrm{~N}=46, \sigma=0.13(\mathrm{LMC}) \\
\pm .03
\end{gathered}
$$

$$
\begin{aligned}
& <\mathrm{Ho}>=-3.25 \mathrm{~d} \log \mathrm{P}+13.30, \mathrm{~N}=83, \sigma=0.18(\mathrm{SMC}) \text {. } \\
& \pm .07 \quad \pm .03
\end{aligned}
$$

A geometrical tilt has once again been permitted to optimize the residuals; it is consistent with the PLC tilt values.

Visvanathan $(1985,1989)$ and Mathewson et al. $(1986,1988)$ have published Iv-band $(1.05 \mu \mathrm{m})$ data on LMC and SMC Cepheids, based on few-phase data corrected to mean light. The SMC data are purposely concentrated in the central bar region alone (i.e. the optically bright core SMC). CL plot these PL-Iv relations in the Clouds:

and

$$
\begin{aligned}
& <\text { Ivo }>=-3.01 \mathrm{~d} \log \mathrm{P}+13.40, \mathrm{~N}=19, \sigma=0.08(\mathrm{LMC}) \\
& \pm .05 \pm .02
\end{aligned}
$$

$$
\begin{gathered}
<\mathrm{I} 0>=-2.92 \mathrm{~d} \log \mathrm{P}+13.97, \mathrm{~N}=85, \sigma=0.20(\mathrm{SMC}) . \\
\pm .07 \quad \pm .02
\end{gathered}
$$

Because both data sets have a quite limited angular coverage of the Clouds, the global tilt corrections from the PLC solution above were used first to adjust the magnitudes. The scatter of the LMC Iv points is remarkably small. If one solves for the B-V period-colour relation for the same sample of 19 stars, the colour scatter is only 0.04 , less than half the true scatter of the strip in B-V, 0.09. Evidently, the particular selection of 19 Cepheids by chance does not sample the full width of the instability strip in Iv.

In the SMC the 41 Iv-Cepheids which are nominally within the dark cloud zones are $0.13 \pm$ 0.04 dimmer than the 44 lying outside. Since A(Iv) is approximately E(B-V), this may be a fair estimate of the typical excess reddening in these regions. Excluding the "inside" ones from the zeropoint changes it to 13.91 in equation (10).

The SMC Iv data provide an independent view of the SMC depth structure. If one projects the distances implied by these data onto the bar axis defined above, there seems to be satisfactory agreement with the PLC depth picture, granting an arbitrary vertical shift and greater distance modulus scatter per Iv point than per PLC point. We would estimate these uncertainties as about $0.12 \mathrm{mag}$ and $0.07 \mathrm{mag}$, respectively. This is somewhat larger than the estimate of 0.085 distance modulus uncertainty per Iv point, made by Mathewson et al. (1988), but is in better agreement with the known width of the instability strip (see Feast 1989). Both the PLC and Iv data are consistent with the densest part of the SMC being a highly inclined prolate spheroid. There is no 
compelling evidence from the combined Cepheid photometry that the bright core part of the SMC is spatially bifurcated in distance, rather than simply highly inclined and elongated.

\section{The "depth" of the Clouds}

\subsection{LMC}

The most suitable tracer, in light of the sparse Iv data, is the PLC(B-V). The total depth from edge to edge $\left(6.4^{\circ}\right)$ then amounts to about $0.16 \mathrm{mag}$ or about $4 \mathrm{ipc}$ at a distance of $50 \mathrm{kpc}$. There is no compelling evidence for in situ depth, since the observed scatters do not significantly exceed the observational expectation. This accords with the line-of-sight kinematics (Feast 1989).

\subsection{SMC}

One should perhaps make a distinction between the central SMC, i.e. the few degrees encompassing the isophotally bright core, and the extended SMC shown by tracers out to almost $10^{\circ}$. Restricted to the core, the PLC and PL(Iv) concur in finding a depth of about $8 \mathrm{kpc}$. The in situ depth may be even smaller, but because of the high inclination one can roughly characterize the core as a whole by this depth.

Following the wing eastwards from the core, the Cepheid distances steadily decrease (Fig. 4) while, conversely, for several degrees preceding southwest from the core, extremely large distances occur. Thus the global end-to-end depth of all these "bits" of the SMC is indeed large; from the extreme wing to the extreme "back-wing" amounts to $0.70 \mathrm{mag}$ or about $19 \mathrm{kpc}$ at a distance of $59 \mathrm{kpc}$. The Cepheids do not show significant in situ depth in the peripheral regions, with the exception that two groups, well separated in distance, are seen superimposed on the sky southwest of the core. Welch et al. (1987) have estimated a tidal radius of $8 \mathrm{kpc}$ for the SMC. This would imply that both the wing and the "back-wing" are tidally lost to the core SMC selfgravity.

The picture of the SMC just presented is founded solely upon the SMC photometry discussed above. Mathewson et al. (1988) have pioneered a parallel approach of using approximate $( \pm 15$ $\mathrm{km} \mathrm{s}^{-1}$ ) SMC Cepheid radial velocities to identify substructures within the central SMC core, which they delineate based on radial velocity and position similarities. The reality of these structures has, however, been questioned by Feast (1989).

\section{The distance moduli of the Clouds}

Feast and Walker (1987) and Walker (1987a) have reviewed the calibrating Cepheids. We have modified their treatment as follows: 1) drop SU Cas, QZ Nor (overtone), and S Vul, 2) correct the erroneous period of SZ Tau, and 3) add CS Vel (Walker 1987c) and the new analysis of NGC7790 (Romeo et al. 1989). We transformed the B-V colours which feast and Walker obtained from Moffett and Barnes (1985) onto the Johnson system, and have included extensive unpublished results on the infrared colours of the calibrators by Laney along with the results of Welch et al. (1985). A median was taken of the zeropoints (cf. CL) to reduce sensitivity to outliers. The uncertainty of the median zeropoint is about 0.045 . The Cloud distance moduli on a completely homogeneous calibration then are: 


\begin{tabular}{lccccc} 
& \multicolumn{2}{c}{ LMC } & \multicolumn{2}{c}{ SMC } \\
& UNCORR & CORR & UNCORR & CORR & NCALIB \\
PL(V) & 18.56 & 18.55 & 18.82 & 18.80 & 26 \\
PLC & 18.69 & 18.52 & 19.36 & 18.92 & 26 \\
PL(H) & 18.51 & 18.48 & 18.98 & 18.88 & 21
\end{tabular}

The metallicity effect corrections were estimated for the visual relations using Stothers' (1988) formalism with the abundances from Caldwell and Coulson (1986), and for the infrared from Johnson's (1966) tabulation of the metallicity effect on the bolometric correction to H. The PLCgeometrical correction in distance modulus from the centroid positions of the Cepheids to the conventional Cloud centres (de Vaucouleurs \& Freeman 1972) is +0.02 in the LMC and +0.03 in the SMC.

\section{References}

Caldwell, J.A.R., Coulson, I.M. (1984a), S.A.A.O. Circ. 8, 1.

Caldwell, J.A.R., Coulson, I.M. (1984b), S.A.A.O. Circ. 9, (i).

Caldwell, J.A.R., Coulson, I.M. (1985a), M.N.R.A.S. 212, 879.

Caldwell, J.A.R., Coulson, I.M. (1985b), M.N.R.A.S. 214, 639.

Caldwell, J.A.R., Coulson, I.M. (1986), M.N.R.A.S. 218, 223.

Caldwell, J.A.R., Coulson, I.M., Spencer Jones, J.H., Black, C.A, Feast, M.W. (1986), M.N.R.A.S. 220, 671.

Caldwell, J.A.R., Laney, C.D. (1990), S.A.A.O. Circ. in prep. (CL).

Dean, J.F. (1981), S.A.A.O. Circ. 6, 10.

de Vaucouleurs, G., Freeman, K.C. (1972), Vistas Astron. 14, 163.

Feast, M.W. (1989), Recent Developments in Magellanic Cloud Research, K.S. de Boer, F. Spite, G. Stasinska (eds.) (Observatoire de Paris: Paris) p.75.

Feast, M.W., Walker, A.R. (1987), Ann. Rev. Astron. Ap. 25, 345.

Freedman, W.L., Grieve, G.R., Madore, B.F. (1985), Ap. J. Suppl. 59, 311.

Glass, I.S. (1985), Irish Astron. J. 17, 1.

Grieve, G.R., Madore, B.F. (1986), Ap. J. Suppl. 62, 427.

Harris, H.C. (1981), Astron. J. 86, 1192.

Harris, H.C. (1983), Astron. J. 88, 507.

Hodge, P.W. (1972), Publ. astr. Soc. Pacific 84, 365.

Hodge, P.W. (1974), Publ. astr. Soc. Pacific 86, 263.

Hodge, P.W., Lee, S.-O. (1984), Ap. J. 276, 509.

Johnson, H.L. (1966), Ann. Rev. Astron. Ap. 4, 193.

Kurochkin, N.W., Tokovinin, A.A., Loggins. A. (1989), Inf. Bull. Variable Stars 3365.

Laney, C.D., Stobie, R.S. (1986a), S.A.A.O. Circ. 10, 51.

Laney, C.D., Stobie, R.S. (1986b), M.N.R.A.S. 222, 449.

Madore, B.F. (1985), IAU Colloq. 82 Cepheids: Theory and Observations, B.F. Madore (ed.) p.157.

Martin, W.L. (1980), S.A.A.O. Circ. 5, 172.

Martin, W.L. (1981), S.A.A.O. Circ. 6, 96.

Martin, W.L., Thomas, Y.Z.R., Carter, B.S., Davies, H.E. (1981), S.A.A.O.Circ. 6, 31.

Mateo, M., Olszewski, E., Madore, B.F. (1990), Ap. J. 353, L11.

Mathewson, D.S., Ford, V.L., Visvanathan, N. (1986), Ap. J. 301, 664.

Mathewson, D.S., Ford, V.L., Visvanathan, N. (1988), Ap. J. 333, 617. 
Moffett, T., Barnes, T. (1985), Ap. J. Suppl. 58, 843.

Payne-Gaposchkin, C. (1971), Smithsonian Contrib. Ap. 13.

Payne-Gaposchkin, C., Gaposchkin, S. (1966), Smithsonian Contrib. Ap. 9.

Romeo, G., Bonifazi, A., Fusi Pecci, F., Tosi, M. (1989), M.N.R.A.S. 240, 459.

Stothers, R.B. (1988), Ap. J. 329, 712.

van Genderen, A.M. (1983a), Astron. Ap. Suppl. 52, 423.

van Genderen, A.M. (1983b), Astron. Ap. 119, 192.

van Genderen, A.M., Hadiyanto Nitihardjo, G. (1989), Astron. Ap. 221, 230.

Visvanathan, N. (1985), Ap. J. 288, 182.

Visvanathan, N. (1989), Ap. J. 346, 629.

Walker, A.R. (1987a), S.A.A.O. Circ. 11, 131.

Walker, A.R. (1987b), M.N.R.A.S. 225, 627.

Walker, A.R. (1987c), M.N.R.A.S. 229, 31.

Wayman, P.A., Stift, M.J., Butler, C.J. (1984), Astron. Ap. Suppl. 56, 169.

Welch, D.L., McAlaray, C.W., Madore, B.F., McLaren, R.A., Neugebauer, G. (1985), Ap. J. 292, 217.

Welch, D.L., McLaren, R.A., Madore, B.F., McAlary, C.W. (1987), Ap. J. 321, 162.

Wesselink, A.J. (1959), M.N.R.A.S. 119, 576. 